\title{
UM NOVO ALGORITMO PARA A MODELAGEM DINÂMICA DE MANIPULADORES FLEXÍVEIS
}

\author{
Celiane C. Machado* \\ mmaccmefurg.br \\ Sebastião C. P. Gomes* \\ dmtscpg@furg.br
}

\author{
Adriana E. L. Pereira* \\ mmaaelp@furg.br \\ Álvaro L. de Bortoli ${ }^{\dagger}$ \\ dbortoli@mat.ufrgs.br
}

\begin{abstract}
*Departamento de Matemática, Fundação Universidade Federal do Rio Grande, Cx. P. 474, Av. Itália Km 8, 96201-900, Rio Grande, RS, BRASIL

${ }^{\dagger}$ PPGMAp, Universidade Federal do Rio Grande do Sul, Cx. P. 15091, 91509-900, Porto Alegre, RS, BRASIL
\end{abstract}

\begin{abstract}
Active control law development of flexible structures is still an open problem. It has been the object of many researches, mainly starting from the eighties. In spite of many works dedicated to the dynamic modeling of such structures, the obtaining of a realistic dynamic model is of fundamental importance for the control law development. In the present work it is introduced a new algorithm to determinate in a quite simple way, the dynamic differential equations of a one flexible link manipulator. It is still developed analytic transfer functions for this specific problem, which are important for the validation of the lumped mass approach model proposed in the form of an algorithm.
\end{abstract}

KEYWORDS: Flexible structures, flexible robots modelling, lumped mass modelling approach.

\section{RESUMO}

Controlar de forma ativa estruturas flexíveis permanece um problema ainda em aberto, tendo sido objeto de muitas pesquisas, principalmente a partir dos anos oitenta. Apesar de existirem muitos trabalhos dedicados à modelagem dinâmica de tais estruturas, a obtenção de um modelo dinâmico realista

Artigo submetido em 20/12/00

1a. Revisão em 24/09/01

Aceito sob recomendação do Ed. Assoc. Prof. Paulo E. Miyagi e preditivo é de fundamental importância para o desenvolvimento de controladores. No presente artigo propõe-se um novo algoritmo para determinar de forma bastante simples, as equações diferenciais da dinâmica de uma estrutura do tipo manipulador com um único elo flexível. Desenvolve-se ainda funções de transferência analíticas para este problema específico, as quais são importantes para a validação do modelo discreto proposto na forma de um algoritmo.

PALAVRAS-CHAVE: Estruturas flexíveis, modelagem de robôs flexíveis, modelagem por aproximação discreta.

\section{INTRODUÇÃO}

O controle de estruturas flexíveis tem sido objeto de diversos estudos realizados principalmente a partir dos anos oitenta (Moorehead e Wang,1997), (Talebi et alli,1999). A maioria os artigos trata de aspectos vinculados à teoria de controle (Machado,1999). Porém, o conhecimento de um modelo dinâmico bem representativo da realidade do fenômeno é de fundamental importância para o projeto de controladores com chances reais de funcionarem na prática. Aplicações de controle de estruturas flexíveis têm sido mais canalizadas para robôs manipuladores com elos flexíveis (Schmitz,1985). Em geral, o método dos modos assumidos (Soares,1997) tem sido mais empregado para a obtenção do modelo dinâmico, principalmente no caso de um único elo flexível. Porém, se o manipulador possui mais de um elo com flexibilidade 
importante, esta técnica de modelagem dinâmica torna-se difícil de ser utilizada em razão de complicações advindas das condições de contorno (Pereira,1999). Portanto, tentase, neste artigo, resgatar o formalismo discreto (lumped mass approach ) (Gomes e Chrétien,1992), o qual possui como grande vantagem a simplicidade de equacionamento. Apesar de apresentar concepção simples, o formalismo discreto é trabalhoso, principalmente se o modelo tiver mais de dois modos flexíveis. Propõe-se então, neste artigo, um algoritmo que simplifica totalmente a obtenção do modelo para qualquer que seja o número de modos flexíveis considerados, além de introduzir modificações, em relação ao formalismo discreto original, que aumentam a precisão do modelo discreto. Apresenta-se, ainda no presente trabalho, um estudo analítico, o qual foi de fundamental importância à validação do modelo discreto estudado.

\section{ESTUDO ANALÍTICO}

Considera-se, inicialmente, uma estrutura flexível de comprimento $l$, conforme ilustrada na figura 1 , articulada em uma extremidade a partir do rotor do atuador, no qual é aplicado um torque motor $T_{m}$, sendo livre na outra extremidade na qual é colocada uma carga. $I_{r}$ é a inércia do rotor e $m_{c}$ a massa da carga (de momento de inércia rotacional desprezível).

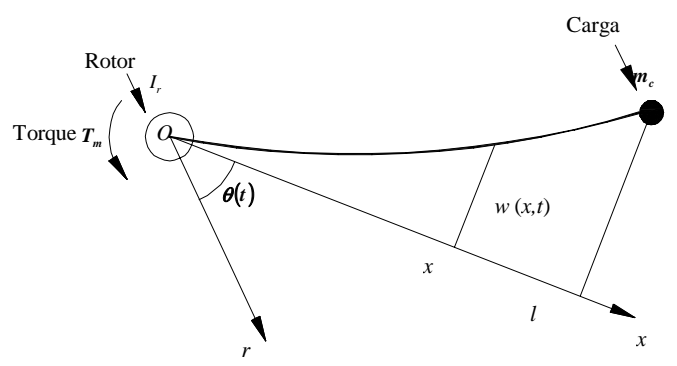

Figura 1: Estrutura flexível.

\subsection{Equações da Dinâmica}

As equações das Energias Cinética e Potencial são dadas por Pereira (1999):

$$
\begin{gathered}
E_{c}=\frac{1}{2} I_{r} \dot{\theta}^{2}+\frac{1}{2} \int_{0}^{l}\left(\frac{\partial w}{\partial t}+x \dot{\theta}\right)^{2} \rho d x+\left.\frac{m_{c}}{2}\left(\frac{\partial w}{\partial t}+x \dot{\theta}\right)^{2}\right|_{x=l} \\
E_{p}=\frac{1}{2} \int_{0}^{l} E I\left(\frac{\partial^{2} w}{\partial x^{2}}(x, t)\right)^{2} d x
\end{gathered}
$$

onde $\rho$ é a massa por unidade de comprimento da linha elástica, $E$ é o Módulo d'Young e $I$ é o momento de inércia da área da secção transversal da estrutura.

Aplicando-se o Princípio de Hamilton e considerando-se a transformação de variável sugerida por Brakwel (Sch- mitz,1985)

$$
y(x, t)=w(x, t)+x \theta(t),
$$

obtém-se o sistema de equações:

$$
\begin{gathered}
E I \frac{\partial^{4} y}{\partial x^{4}}(x, t)+\rho \frac{\partial^{2} y}{\partial t^{2}}(x, t)=0, \\
I_{r} \ddot{\theta}(t)+\rho \int_{0}^{l} x \frac{\partial^{2} y}{\partial t^{2}} d x+m_{c} l \ddot{y}(l, t)=T_{m},
\end{gathered}
$$

com as seguintes condições de contorno:

$$
\begin{aligned}
& \left.E I \frac{\partial^{2} y}{\partial x^{2}}(x, t)\right|_{x=0}+T_{m}-I_{r} \ddot{\theta}=0 \\
& \left.y(x, t)\right|_{x=0}=y(0, t)=w(0, t)+0 . \theta(t)=0 \\
& \left.E I \frac{\partial^{2} y}{\partial x^{2}}(x, t)\right|_{x=l}=E I \frac{\partial^{2} w}{\partial x^{2}}(l, t)=0 \\
& \left.E I \frac{\partial^{3} y}{\partial x^{3}}(x, t)\right|_{x=l}=\left.m_{c} \frac{\partial^{2} y}{\partial t^{2}}(x, t)\right|_{x=l}
\end{aligned}
$$

Aplicando-se a Transformada de Laplace na equação (1) resulta em:

$$
\frac{d^{4} \bar{y}}{d x^{4}}-\beta^{4} \bar{y}=0
$$

sendo $\beta^{4}=-\frac{\rho}{E I} s^{2}$, com as respectivas condições de contorno:

$$
\begin{aligned}
& \bar{y}(0, s)=0 \\
& \left.E I \frac{d^{2} \bar{y}}{d x^{2}}\right|_{x=0}+\bar{T}(s)=\left.I_{r} s^{2} \frac{d \bar{y}}{d x}\right|_{x=0} \\
& \left.E I \frac{d^{2} \bar{y}}{d x^{2}}\right|_{x=l}=0 \\
& \left.E I \frac{d^{3} \bar{y}}{d x^{3}}\right|_{x=l}=\left.m_{c} s^{2} \bar{y}\right|_{x=l}
\end{aligned}
$$

onde $\bar{T}(s)$ é a transformada de Laplace de $T_{m}(t)$. A equação (2) admite solução geral na forma:

$$
\begin{aligned}
\bar{y}=A \operatorname{sen}(\beta x)+B & \operatorname{senh}(\beta x)+ \\
& +C \cos (\beta x)+D \cosh (\beta x) .
\end{aligned}
$$

Pode-se comprovar ainda que (Pereira, 1999):

$$
\bar{\theta}(s)=\frac{\partial \bar{y}}{\partial x}(0, s)
$$

Definindo-se $\lambda=\beta l$ e aplicando-se as condições de contorno (3) à equação (4) resulta no sistema:

$$
M(\lambda)\left[\begin{array}{l}
A \\
B \\
C
\end{array}\right]=-\frac{1}{E I \beta^{2}}\left[\begin{array}{l}
\bar{T} \\
0 \\
0
\end{array}\right],
$$

onde:

$$
\begin{aligned}
& M(\lambda)= \\
& {\left[\begin{array}{ccc}
\varepsilon \lambda^{3} & \varepsilon \lambda^{3} & -2 \\
-s \lambda & s h \lambda & -(c \lambda+\operatorname{ch} \lambda) \\
-(c \lambda+\eta \lambda s \lambda) & c h \lambda+\eta \lambda \operatorname{sh} \lambda & s \lambda-\operatorname{sh} \lambda+\eta \lambda(c \lambda-\operatorname{ch} \lambda)
\end{array}\right]}
\end{aligned}
$$


e

$$
\begin{gathered}
s \lambda=\operatorname{sen}(\lambda) ; \operatorname{sh}(\lambda)=\operatorname{senh}(\lambda) ; \\
c \lambda=\cos (\lambda) ; \operatorname{ch} \lambda=\cosh (\lambda) ; \\
D=-C ; \varepsilon=\frac{I_{r}}{3 I_{B}} \text { e } \eta=\frac{m_{c} l^{2}}{3 I_{B}},
\end{gathered}
$$

sendo $I_{r}$ o momento de inércia do rotor e $I_{B}$ o momento de inércia da lâmina. As constantes $A, B, C$ e $D$ podem ser obtidas da solução da equação (6) e são funções de $\lambda$ e de $\bar{T}(s)$. Substituindo-as na solução geral (4), obtém-se $\bar{y}(s)$ em função de $\lambda$ e de $\bar{T}(s)$.

\subsection{Funções de Transferência Analíticas}

Os pólos das funções de transferência, são as raízes da equação $D(\lambda)=0$, sendo $D(\lambda)$ obtido pelo determinante da matriz $M(\lambda)$, que em série de Taylor pode ser escrito como:

$$
\begin{aligned}
& D(\lambda)=\frac{4}{3} \lambda^{3}(1+3 \varepsilon+3 \eta) \prod_{i=0}^{i=\infty}\left(1-\frac{\lambda^{4}}{\lambda_{i}^{4}}\right)= \\
& =\frac{4}{3} \lambda^{3}(1+3 \varepsilon+3 \eta) \prod_{i=1}^{i=\infty}\left(1+\frac{w^{2}}{w_{i}^{2}}\right)
\end{aligned}
$$

sendo $w_{i}=\sqrt{\frac{E I}{\rho l^{4}}} \lambda_{i}^{2}, i=1,2, \ldots, \infty$ e $\lambda_{i}, i=1,2, \ldots \infty$ as raízes de $D(\lambda)=0$.

As equações (3), (5) e (6) permitem obter o numerador da função de transferências do caso colocado, enquanto que as equações (3), (4) e (6) permitem obter o numerador no caso não colocado (Pereira,1999). No caso colocado, observase a posição angular do rotor, ou seja, a observação é feita no mesmo local no qual está sendo aplicado o torque motor. Neste caso, a função de transferência apresenta-se na forma:

$$
\frac{\bar{\theta}(s)}{\bar{T}(s)}=\frac{N_{\theta}(\lambda)}{D(\lambda)}=\frac{1}{I_{T} s^{2}} \prod_{i=1}^{i=\infty} \frac{\left(1+\frac{s^{2}}{\Omega_{i}^{2}}\right)}{\left(1+\frac{s^{2}}{w_{i}^{2}}\right)},
$$

onde $I_{T}=I_{B}+I_{r}+m_{c} l^{2}$ é o momento de inércia de corpo rígido. O gráfico desta função de transferência pode ser observado na figura 2, na qual destacam-se os pólos (picos para mais infinito) e zeros (picos para menos infinito), denotando assim que o sistema é de fase mínima.

No caso não colocado, conforme mostra a figura 3, observase a posição da carga, ou seja, a observação é feita numa extremidade, enquanto que o torque motor é aplicado na outra extremidade, existindo portanto toda uma dinâmica flexível entre o local da aplicação do torque e o da realização da observação. Neste caso, a função de transferência apresentase na forma:

$$
\frac{\bar{y}_{t}(s)}{\bar{T}(s)}=\frac{N_{y t}(\lambda)}{D(\lambda)}=\frac{l}{I_{T} s^{2}} \prod_{i=1}^{i=\infty} \frac{\left(1-\frac{s^{2}}{\alpha_{i}^{2}}\right)}{\left(1+\frac{s^{2}}{w_{i}^{2}}\right)}
$$

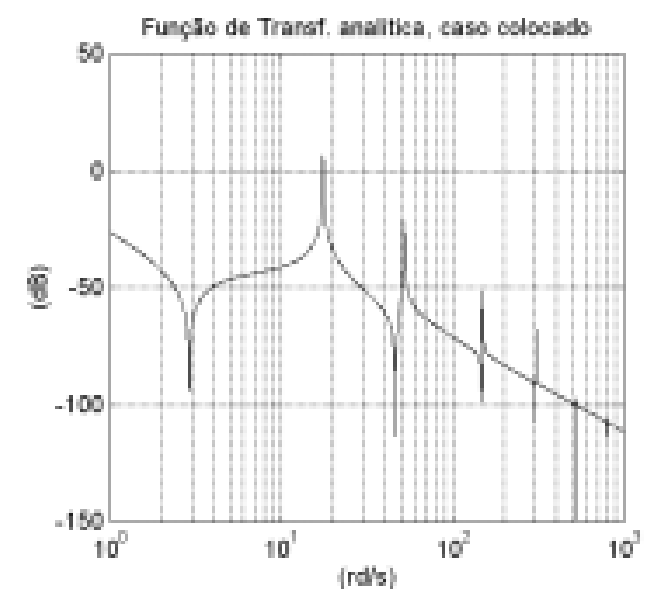

Figura 2: Gráfico da Função de Transferência Analítica (caso colocado).

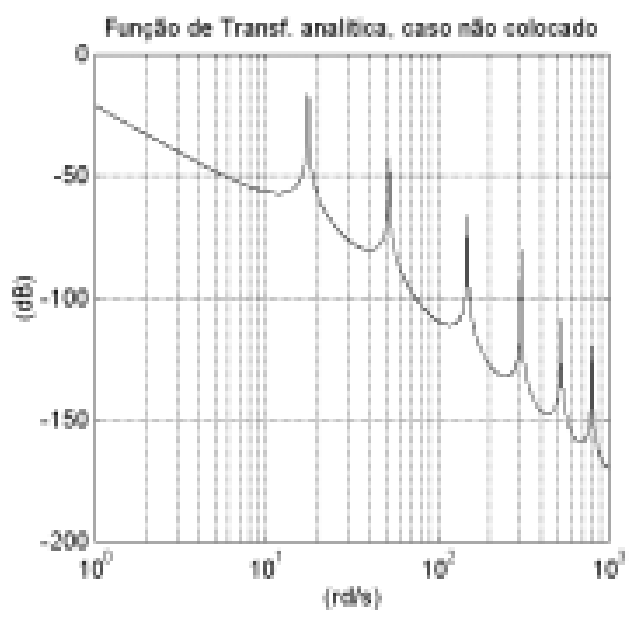

Figura 3: Gráfico da Função de Transferência Analítica (caso não colocado).

\section{MODELO DA ESTRUTURA UTILI- ZANDO O FORMALISMO DISCRETO}

O procedimento de modelagem apresentado a seguir é denominado formalismo discreto (lumped mass approach), o qual representa a flexibilidade contínua por uma aproximação discreta, a partir da introdução de articulações fictícias (Gomes e Chrétien, 1992).

A modelagem pode ser obtida a partir do número de articulações fictícias desejado. Estas articulações são posicionadas na estrutura como mostra a figura 4. Para o caso de uma única articulação fictícia, divide-se a estrutura em duas partes de mesmo comprimento (elementos rígidos) e coloca-se a articulação entre elas; para o modelo com duas articulações fictícias, cada articulação é posicionada na me- 
tade de cada elemento rígido em que a estrutura foi dividida no caso anterior e para $n$ articulações fictícias, as articulações são posicionadas na metade de cada elemento rígido do caso de $n-1$ articulações fictícias.

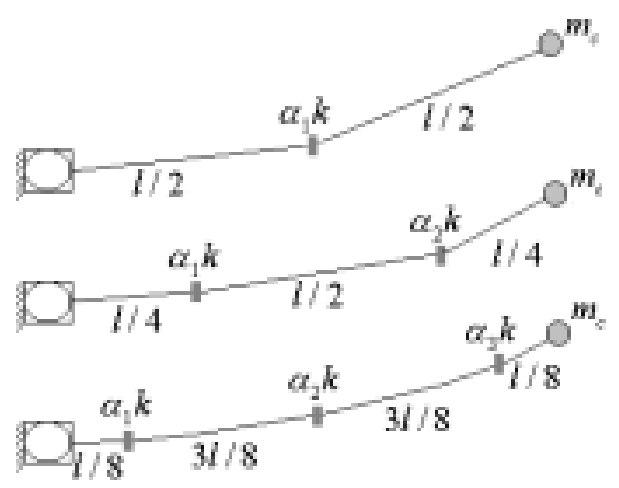

Figura 4: Estrutura Flexível e sua aproximação discreta para os casos de uma, duas e três articulações fictícias.

Depois de posicionadas as articulações fictícias e supondo $l$ o comprimento da estrutura, conhecendo-se as massas de cada elemento rígido (concentradas em seus respectivos centros de massa), a massa da carga terminal e as constantes elásticas de cada articulação fictícia, considerando-se $\theta_{i}$ o ângulo entre a direção $x$ e o respectivo elemento rígido de número $i$, obtém-se as energias cinética e potencial e consequentemente o Lagrageano do sistema. Por meio das equações de Euler-Lagrange e ainda, considerando-se pequenas deformações angulares nas articulações fictícias, obtémse o modelo dinâmico:

$$
[I] \ddot{\theta}+\left[C_{a t}\right] \dot{\theta}+\left[K_{e l}\right] \theta=B^{\prime} T_{m}
$$

sendo $n$ o número de articulações fictícias, $[I]_{n+1, n+1}$ a matriz de inércia, $\left[K_{e l}\right]_{n+1, n+1}$ a matriz de constantes elásticas, $\left[C_{a t}\right]_{n+1, n+1}$ a matriz de atritos e $B^{\prime}=$ $\left[\begin{array}{llll}1 & 0 & \cdots & 0\end{array}\right]^{T}$.

\section{ALGORITMO PARA OBTENÇÃO DO MODELO DINÂMICO CONSIDERANDO N ARTICULAÇÕES FICTÍCIAS}

Define-se inicialmente os vetores $p, C, m$ e $K$ utilizados no algoritmo proposto neste trabalho. $\mathrm{O}$ vetor $p$ (vetor de comprimentos dos elementos rígidos ) é obtido a partir do número de articulações fictícias e do comprimento da estrutura considerado. Por exemplo, para duas articulações fictícias, seus elementos são: $p(1)=l / 4, p(2)=l / 2$ e $p(3)=l / 4$, conforme mostra a figura 4 . O vetor $m$ (vetor de massas dos elementos rígidos) é obtido por meio da expressão $m(i)=\frac{m_{b} p(i)}{l}$, sendo $m_{b}$ a massa da estrutura e $i=1, \ldots, n+1$, onde $n$ é o número de articulações fictícias. Define-se os elementos do vetor $K$ (constantes elásticas das articulações fictícias) na forma: $k_{j}=\alpha_{j} k=\alpha_{j} \frac{n E I}{l}$, onde $E I$ é o Módulo d'Young vezes a inércia da secção reta, $j=1, \ldots, n$ e $\alpha_{j}$ é uma constante que multiplica $k$ a fim de minimizar os erros nas freqüências dos modos de vibração.

\subsection{Descrição do Algoritmo}

Conforme será mostrado na seqüência, apenas a matriz de inércia merece ser posta na forma algoritmica, uma vez que as matrizes de atrito e de constantes elásticas possuem montagem evidente em função do número de articulações fictícias consideradas. A obtenção da matriz $[I]_{n+1, n+1}$ obedece então aos passos apresentados a seguir.

Supondo-se $i=1, \ldots, n+1$ :

(Diagonal Principal)

Se $i=1$

$$
I_{i, i}=I r+p(i)^{2} \times\left(\frac{m(i)}{4}+m_{c}+\sum_{z=i+1}^{n+1} m(z)\right)
$$

Se $i \neq 1$

$$
I_{i, i}=p(i)^{2} \times\left(\frac{m(i)}{4}+m_{c}+\sum_{z=i+1}^{n+1} m(z)\right)
$$

(Acima da Diagonal Principal)

$$
I_{i, j}=p(j) \times p(i) \times\left(\frac{m(j)}{2}+m_{c}+\sum_{z=j+1}^{n+1} m(z)\right)
$$

(Abaixo da Diagonal Principal)

$$
I_{i, j}=I_{j, i}
$$

\section{EXEMPLOS DE APLICAÇÃO DO ALGO- RITMO E OBTENÇÃO DE MODELOS}

Qualquer que seja o número de articulações fictícias a ser considerado, o importante é posicioná-las conforme verificado na figura 4 e posteriormente, obter os elementos dos vetores $p, m$ e $K$ de acordo com o especificado no início da seção 4. A matriz de coeficientes de atritos pode ser identificada a partir de experimentos em malha aberta, uma vez que a mesma depende fundamentalmente do material e das dimensões da estrutura. 


\subsection{Exemplo Fictícia}

$$
\begin{gathered}
p(1)=l / 2 \text { e } p(2)=l / 2 \\
{[I]=\left[\begin{array}{cc}
I r+\frac{l^{2}}{4}\left(\frac{m_{1}}{4}+m_{c}+m_{2}\right) & \frac{l^{2}}{4}\left(\frac{m_{2}}{2}+m_{c}\right) \\
\frac{l^{2}}{4}\left(\frac{m_{2}}{2}+m_{c}\right) & \frac{l^{2}}{4}\left(\frac{m_{2}}{4}+m_{c}\right)
\end{array}\right]} \\
{\left[C_{a t}\right]=\left[\begin{array}{ll}
c_{r}+c_{1} & -c_{1} \\
-c_{1} & c_{1}
\end{array}\right]}
\end{gathered}
$$

e

$$
\left[K_{e l}\right]=\left[\begin{array}{ll}
\alpha_{1} k & -\alpha_{1} k \\
-\alpha_{1} k & \alpha_{1} k
\end{array}\right],
$$

onde $c_{r}$ é o coeficiente de atrito viscoso atuante no rotor e $k=\frac{n E I}{l}, \operatorname{com} n=1$.

\subsection{Exemplo para Duas Articulações Fictícias}

$p(1)=l / 4, p(2)=l / 2$ e $p(3)=l / 4$

$$
\begin{gathered}
{[I]=\left[\begin{array}{ccc}
I_{11} & I_{12} & I_{13} \\
I_{21} & I_{22} & I_{23} \\
I_{31} & I_{32} & I_{33}
\end{array}\right]} \\
I_{11}=I r+\frac{l^{2}}{16}\left(\frac{m_{1}}{4}+m_{c}+m_{2}+m_{3}\right), \\
I_{21}=\frac{l^{2}}{8}\left(\frac{m_{2}}{2}+m_{c}+m_{3}\right), \\
I_{31}=\frac{l^{2}}{16}\left(\frac{m_{3}}{2}+m_{c}\right), \quad I_{21}=I_{12}, \\
I_{22}=\frac{l^{2}}{4}\left(\frac{m_{2}}{4}+m_{c}+m_{3}\right), \\
I_{23}=\frac{l^{2}}{8}\left(\frac{m_{3}}{2}+m_{c}\right), \quad I_{31}=I_{13}, \quad I_{32}=I_{23}
\end{gathered}
$$

$\mathrm{e}$

$$
\begin{gathered}
I_{33}=\frac{l^{2}}{16}\left(\frac{m_{3}}{4}+m_{c}\right) \\
{\left[C_{a t}\right]=\left[\begin{array}{lll}
c_{r}+c_{1} & -c_{1} & 0 \\
-c_{1} & c_{1}+c_{2} & -c_{2} \\
0 & -c_{2} & c_{2}
\end{array}\right]}
\end{gathered}
$$

$\mathrm{e}$

$$
\left[K_{e l}\right]=\left[\begin{array}{lll}
\alpha_{1} k & -\alpha_{1} k & 0 \\
-\alpha_{1} k & \alpha_{1} k+\alpha_{2} k & -\alpha_{2} k \\
0 & -\alpha_{2} k & \alpha_{2} k
\end{array}\right]
$$

onde $k=\frac{n E I}{l}$, com $n=2$.

\subsection{Exemplo para Três Articulações Fictícias}

$p(1)=l / 8, p(2)=3 l / 8, p(3)=3 l / 8$ e $p(4)=l / 8$

$$
[I]=\left[\begin{array}{llll}
I_{11} & I_{12} & I_{13} & I_{14} \\
I_{21} & I_{22} & I_{23} & I_{24} \\
I_{31} & I_{32} & I_{33} & I_{34} \\
I_{41} & I_{42} & I_{43} & I_{44}
\end{array}\right]
$$

onde

$$
\begin{aligned}
& I_{11}=I r+\frac{l^{2}}{64}\left(\frac{m_{1}}{4}+m_{c}+m_{2}+m_{3}+m_{4}\right), \\
& I_{12}=\frac{3 l^{2}}{64}\left(\frac{m_{2}}{2}+m_{c}+m_{3}+m_{4}\right) \text {, } \\
& I_{13}=\frac{3 l^{2}}{64}\left(\frac{m_{3}}{2}+m_{c}+m_{4}\right), \\
& I_{14}=\frac{l^{2}}{64}\left(\frac{m_{4}}{2}+m_{c}\right), \quad I_{21}=I_{12} \\
& I_{22}=\frac{9 l^{2}}{64}\left(\frac{m_{2}}{4}+m_{c}+m_{3}+m_{4}\right) \text {, } \\
& I_{23}=\frac{9 l^{2}}{64}\left(\frac{m_{3}}{2}+m_{c}+m_{4}\right), \\
& I_{24}=\frac{3 l^{2}}{64}\left(\frac{m_{4}}{2}+m_{c}\right), \quad I_{31}=I_{13}, \quad I_{32}=I_{23} \text {, } \\
& I_{33}=\frac{9 l^{2}}{64}\left(\frac{m_{3}}{4}+m_{c}+m_{4}\right) \\
& I_{34}=\frac{3 l^{2}}{64}\left(\frac{m_{4}}{2}+m_{c}\right), \quad I_{14}=I_{41}, \quad I_{42}=I_{24} \text {, } \\
& I_{43}=I_{34}, \quad I_{44}=\frac{3 l^{2}}{64}\left(\frac{m_{4}}{2}+m_{c}\right) \text {. } \\
& {\left[C_{a t}\right]=\left[\begin{array}{cccc}
c_{r}+c_{1} & -c_{1} & 0 & 0 \\
-c_{1} & c_{1}+c_{2} & -c_{2} & 0 \\
0 & -c_{2} & c_{2}+c_{3} & -c_{3} \\
0 & 0 & -c_{3} & c_{3}
\end{array}\right]}
\end{aligned}
$$

$\mathrm{e}$

$$
\left[K_{e l}\right]=\left[\begin{array}{cccc}
\alpha_{1} k & -\alpha_{1} k & 0 & 0 \\
-\alpha_{1} k & \alpha_{1} k+\alpha_{2} k & -\alpha_{2} k & 0 \\
0 & -\alpha_{2} k_{2} & \alpha_{2} k+\alpha_{2} k & -\alpha_{3} k \\
0 & 0 & -\alpha_{2} k & \alpha_{3} k
\end{array}\right]
$$

onde $k=\frac{n E I}{l}, \operatorname{com} n=3$. Os multiplicadores $\alpha_{j}(j=1, \ldots$, $n$ ), foram obtidos atribuindo-se valores aos mesmos, entre um valor inicial (próximo de zero) e um final (próximo de 5), com um passo de variação de 0.005. Para cada conjunto de valores $\alpha_{j}$, as frequiências de vibração do modelo discreto eram obtidas e comparadas às frequiências analíticas. Para $n=3$, encontraram-se os seguintes valores os quais possibilitaram erros mínimos nos modos de vibração:

$$
\alpha_{1}=2.645 ; \quad \alpha_{2}=0.78 \quad ; \quad \alpha_{3}=0.145 .
$$




\section{COMPARAÇÕES COM AS FUNÇÕES DE TRANSFERÊNCIA ANALÍTICA}

Negligenciou-se os atritos a fim de possibilitar a comparação com o caso analítico, cujo sistema é conservativo. A figura 5 mostra uma comparação entre as respostas em frequiência analítica, conforme ilustrado na figura 2 , e do modelo discreto considerando-se três articulações fictícias, para o caso colocado. Este resultado mostrou erros menores que $1 \%$ nas frequiências dos modos de vibração.

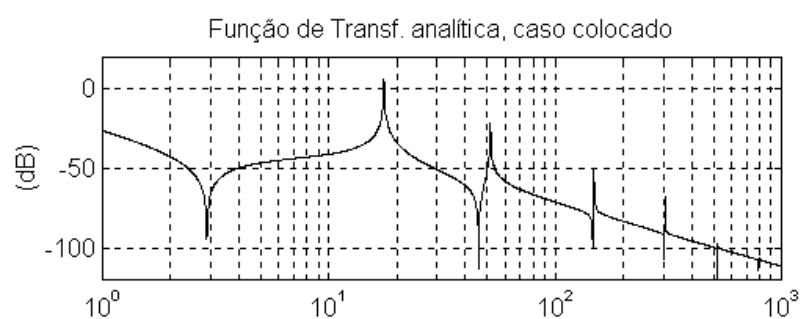

Funçẵo de Transf. Mod. Discreto, caso colocado (3 modos flexíveis)

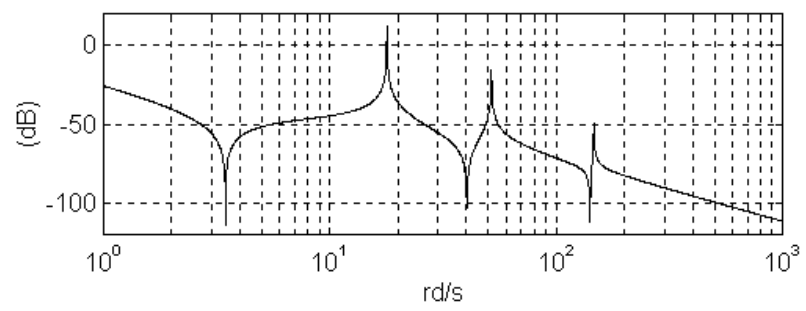

Figura 5: Funções de Transferência Analítica e do Modelo Discreto com 3 modos flexíveis (caso colocado-3af), sendo negligenciados os torques de atrito do Modelo Discreto.

A figura 6 mostra uma comparação entre as respostas em frequiência analítica, como ilustrado na figura 2, e do modelo discreto considerando-se quatro articulações fictícias, para o caso colocado. Neste caso, os parâmetros $\alpha_{j}$ que possibilitaram erros mínimos nos modos de vibração foram:

$\alpha_{1}=3.95 ; \quad \alpha_{2}=0.755 \quad ; \quad \alpha_{3}=0.34 ; \quad \alpha_{4}=0.08$

Este resultado mostrou erros também pequenos nas frequiências dos três primeiros modos, mas não se conseguiu um conjunto de parâmetros que possibilitasse erros menores do que $10 \%$ para a frequiência do quarto modo.

\section{CONCLUSÕES}

Sobre o Estudo Analítico Desenvolveu-se as chamadas funções de transferência analíticas, para os casos colocado e não colocado. É importante ressaltar que:

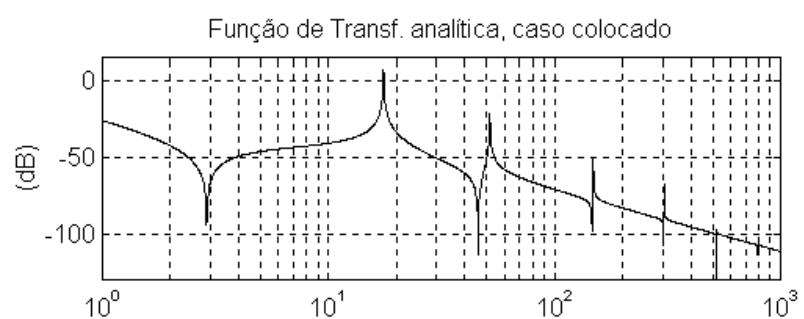

Funçăo de Transf. Mod. Discreto, caso colocado (4 modos flexíveis)

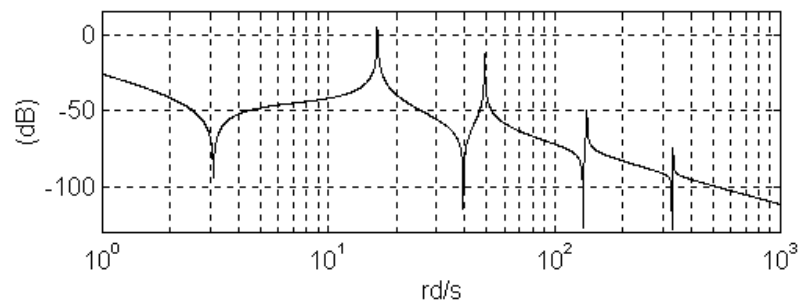

Figura 6: Funções de Transferência Analítica e do Modelo Discreto com 4 modos flexíveis (caso colocado-4af), sendo negligenciados os torques de atrito do Modelo Discreto.

Existe uma hipótese simplificadora na origem do equacionamento, consistindo na consideração de que não há esforços de cizalhamento na estrutura quando esta se deforma;

Estas funções de transferência são exatas e com elas obtémse as respostas freqüenciais as quais revelam os pólos (comum aos casos colocado e não colocado) e os zeros (específicos para cada caso);

Sobre o Formalismo Discreto Esta técnica consiste em aproximar a flexibilidade contínua da estrutura por uma forma discreta, constituída de partes rígidas conectadas por elementos flexíveis chamados de articulações fictícias;

Trata-se da mais simples das técnicas em termos matemáticos uma vez que não há a necessidade de se trabalhar com equações diferenciais parciais, nem, obviamente, com as conseqüentes condições de contorno;

Esta técnica apresentou boa representação de todos os modos de vibração para modelos com até três articulações fictícias $(n=1,2,3)$. Para o caso de quatro articulações fictícias, $\mathrm{n}=4$, conseguiu-se um conjunto de multiplicadores $\alpha_{j}(j=1, \ldots, 4)$ que possibilitou os mesmos pequenos erros, como mostra a figura 6 , nos três primeiros modos, mas com um erro mínimo de $10 \%$ na freqüência do quarto modo;

Os resultados tendem a piorar, caso se deseje um modelo com cinco modos flexíveis ou mais, com erros maiores nos modos de mais alta freqüência.

O formalismo discreto possui grande vantagem com relação 
aos demais, vantagem esta relativa à maior simplicidade na determinação de modelos dinâmicos para estruturas com vários elos flexíveis ou ainda, encontrar os modelos cinemáticos direto e inverso.

\section{REFERÊNCIAS}

Gomes, S. C. P. e J. P. Chrétien (1992). Dynamic modeling and friction compensated control of a 1 d.o.f. rigid or flexible manipulator In Microdynamic et Pointage de Grande Précision, CNES, 1er Symposium Internacional.

Machado, C. C. (1999). Um Estudo Sobre Controle de Estruturas Flexíveis Ativadas por Escoamentos de Fluidos, Dissertação de Mestrado, UFRGS, Porto Alegre - RS, Brasil.

Moorehead, S. J. e D. Wang (1997). An Experimental Study of Contact Transition Control of a Single Flexible Link Using Positive Acceleration Feedback, IEEE-International Conference on Robotics and Automation, Albuquerque, New Mexico.

Pereira, A. E. L. (1999). Um Estudo Sobre Modelagem Matemática de Estruturas Flexíveis, Dissertação de Mestrado, UFRGS, Porto Alegre-RS, Brasil.

Schmitz, E. (1985). Experiments on the End-Point Control of a very Flexible One-link Manipulator, Ph.D. Thesis, Stanford, California, Unite States.

Soares, A. M. S. (1997). Modelagem e identificação experimental de estruturas flexíveis. Tese de Doutorado, ITA, São José dos Campos, Brasil.

Talebi, H. A., K. Khorasani e R. V. Patel (1999). Experimental Results on Tracking Control of a Flexible-Link Manipulator: A New Output Re-definition Approach, IEEE-International Conference on Robotics and Automation, Detroit, Michigan. 